JOURNAL OF THE

CHUNGCHEONG MATHEMATICAL SOCIETY

Volume 26, No. 4, November 2013

http://dx.doi.org/10.14403/jcms.2013.26.4.915

\title{
2-NORM MIDPOINTS AND 2-NORMED EQUALITIES IN 2-NORMED SPACES
}

\author{
SANG-Cho ChUnG*
}

\begin{abstract}
In this paper, we investigate some properties of 2-norm midpoints and 2-normed equalities in 2-normed spaces.
\end{abstract}

\section{Introduction and preliminaries}

We assume that every space is a linear space over the field $\mathbb{R}$ of real numbers.

In the 1960's, the concept of 2-normed spaces was introduced by S. Gähler [1,2] and many mathematicians studied on this subject.

In this paper, under the 2-normed spaces we give easy solutions of Theorem 2.1 of [3] in Theorem 2.3 and investigate some properties of 2-normed equalities in Theorem 2.5.

Let give us some definitions and lemmas for our main results.

Definition 1.1. Let $\mathcal{X}$ be a linear space over $\mathbb{R}$ with $\operatorname{dim} \mathcal{X}>1$ and let $\|\cdot, \cdot\|: \mathcal{X} \times \mathcal{X} \rightarrow \mathbb{R}$ be a function satisfying the following properties: (2N1) $\|x, y\|=0$ if and only if $x$ and $y$ are linearly dependent, (2N2) $\|x, y\|=\|y, x\|$, (2N3) $\|\alpha x, y\|=|\alpha|\|x, y\|$, (2N4) $\|x, y+z\| \leq\|x, y\|+\|x, z\|$

for all $x, y, z \in \mathcal{X}$ and $\alpha \in \mathbb{R}$. Then the mapping $\|\cdot, \cdot\|$ is called a 2norm on $\mathcal{X}$ and the pair $(\mathcal{X},\|\cdot, \cdot\|)$ is called a linear 2-normed space. Sometimes the condition (2N4) called the triangle inequality.

REMARK 1.2. We have some basic properties for a linear 2-normed space $\mathcal{X}$ over $\mathbb{R}$ with $\operatorname{dim} \mathcal{X}>1$.

Received September 31, 2013; Accepted October 30, 2013.

2010 Mathematics Subject Classification: Primary 46B06, 41A65; Secondary 30L99.

Key words and phrases: 2-normed space, 2-metric space, 2-norm midpoint, 2normed equality. 
(1) For all $x, y$ in $\mathcal{X}$, we have $0 \leq\|x, y\|$.

(2) For all $\alpha$ in $\mathbb{R}$ and $x, y$ in $\mathcal{X}$, we have $\|x, y\|=\|x, y+\alpha x\|$.

(3) For all $x, y, z$ in $\mathcal{X}$, we have

$$
|\|x, z\|-\|y, z\|| \leq\|x-y, z\| \leq\|x, z\|+\|y, z\| .
$$

and

$$
|\|x, z\|-\|y, z\|| \leq\|x+y, z\| \leq\|x, z\|+\|y, z\| .
$$

In particular, if $m=\min \{\|x, z\|,\|y, z\|\}$, then

$$
-2 m \leq\|x+y, z\|-\|x-y, z\| \leq 2 m .
$$

Proof. (1) and (2) follow from the definitions of 2-normed spaces.

(3) For all $x, y, z$ in $\mathcal{X}$, we have $\|x, z\|=\|x-y+y, z\| \leq\|x-y, z\|+$ $\|y, z\|$. Hence we have $\|x, z\|-\|y, z\| \leq\|x-y, z\|$.

On the other hand, $\|y, z\|=\|y-x+x, z\| \leq\|y-x, z\|+\|x, z\|$, or $\|y, z\|-\|x, z\| \leq\|y-x, z\|=\|x-y, z\|$. Therefore we get

$$
|\|x, z\|-\|y, z\|| \leq\|x-y, z\| .
$$

The other parts follows from $(2 \mathrm{~N} 2),(2 \mathrm{~N} 3)$ and $(2 \mathrm{~N} 4)$.

Definition 1.3. A sequence $\left\{x_{n}\right\}$ in a linear 2-normed space $\mathcal{X}$ is called a convergent sequence if there is a point $x \in \mathcal{X}$ such that $\lim _{n \rightarrow \infty}\left\|x_{n}-x, y\right\|=0$ for all $y \in \mathcal{X}$. If $\left\{x_{n}\right\}$ converges to $x$, write $x_{n} \rightarrow x$ as $n \rightarrow \infty$ and call $x$ the limit of $\left\{x_{n}\right\}$. In this case, we also write $\lim _{n \rightarrow \infty} x_{n}=x$.

Theorem 1.4. Let $\mathcal{X}$ be a linear 2-normed space with $\operatorname{dim} \mathcal{X}=r$. Suppose that $\left\{x_{n}\right\}$ is a sequence in $\mathcal{X}$ and $\left\{y_{1}, y_{2}, \cdots, y_{r}\right\}$ is a basis of $\mathcal{X}$. Then for a point $x \in \mathcal{X}$ we have the following.

(1) $\lim _{m, n \rightarrow \infty}\left\|x_{n}-x_{m}, y\right\|=0$ for all $y \in \mathcal{X}$ if and only if $\lim _{m, n \rightarrow \infty}\left\|x_{n}-x_{m}, y_{i}\right\|=0$ for $i=1,2, \cdots, r$.

(2) $\lim _{m, n \rightarrow \infty}\left\|x_{n}-x, y\right\|=0$ for all $y \in \mathcal{X}$ if and only if $\lim _{m, n \rightarrow \infty}\left\|x_{n}-x, y_{i}\right\|=0$ for $i=1,2, \cdots, r$.

Proof. (1) $(\Rightarrow)$ It is clear.

$(\Leftarrow)$ For all $y \in \mathcal{X}$, there are numbers $\alpha_{1}, \alpha_{2}, \cdots, \alpha_{r} \in \mathbb{R}$ such that $y=\alpha_{1} y_{1}+\alpha_{2} y_{2}+\cdots+\alpha_{r} y_{r}$. Hence we have

$$
\begin{aligned}
\left\|x_{n}-x_{m}, y\right\| & =\left\|x_{n}-x_{m}, \alpha_{1} y_{1}+\alpha_{2} y_{2}+\cdots+\alpha_{r} y_{r}\right\| \\
& \leq\left\|x_{n}-x_{m}, \alpha_{1} y_{1}\right\|+\cdots+\left\|x_{n}-x_{m}, \alpha_{r} y_{r}\right\| \\
& =\left|\alpha_{1}\right|\left\|x_{n}-x_{m}, y_{1}\right\|+\cdots+\left|\alpha_{r}\right|\left\|x_{n}-x_{m}, y_{r}\right\| .
\end{aligned}
$$

Therefore we have $\lim _{m, n \rightarrow \infty}\left\|x_{n}-x_{m}, y\right\|=0$ for all $y \in \mathcal{X}$.

(2) The proof is similar to (1). 
Lemma 1.5. Let $\mathcal{X}$ be a linear 2-normed space over $\mathbb{R}$ with $\operatorname{dim} \mathcal{X}>1$. Let $\left\{x_{n}\right\}$ be a sequence in $\mathcal{X}$ and $x$ be a vector of $\mathcal{X}$. Then the following are equivalent.

(1) The vector $x$ is a limit of $\left\{x_{n}\right\}$. That is, for all $y$ in $\mathcal{X}$, $\lim _{n \rightarrow \infty}\left\|x_{n}-x, y\right\|=0$.

(2) For all $a, y$ in $\mathcal{X}, \lim _{n \rightarrow \infty}\left\|a-x_{n}, y\right\|=\|a-x, y\|$.

(3) For all $a, y$ in $\mathcal{X}, \lim _{n \rightarrow \infty}\left\|a-x_{n}, y-x_{n}\right\|=\|a-x, y-x\|$.

(4) For all $y$ in $\mathcal{X}, \lim _{n \rightarrow \infty}\left\|x_{n}-y, x-y\right\|=0$.

Proof. (1) $\Rightarrow(2)$ By remark 1.2(3), for all $a, y$ in $\mathcal{X}$, we have the following.

$$
\left|\left\|a-x_{n}, y\right\|-\|a-x, y\|\right| \leq\left\|x_{n}-x, y\right\| .
$$

Hence we have $\lim _{n \rightarrow \infty}\left\|a-x_{n}, y\right\|=\|a-x, y\|$ for all $a, y$ in $\mathcal{X}$.

(2) $\Rightarrow(3)$ For all $a, y$ in $\mathcal{X}$, we have $\lim _{n \rightarrow \infty}\left\|a-x_{n}, y-x_{n}\right\|$ $=\lim _{n \rightarrow \infty}\left\|a-x_{n}, y-a\right\|=\|a-x, y-a\|=\|a-x, y-x\|$.

(3) $\Rightarrow$ (4) Replacing $a$ by $y$ and $y$ by $x$, we have

$$
\lim _{n \rightarrow \infty}\left\|y-x_{n}, x-y\right\|=\lim _{n \rightarrow \infty}\left\|y-x_{n}, x-x_{n}\right\|=\|y-x, x-x\|=0
$$

for all $y$ in $\mathcal{X}$.

$(4) \Rightarrow(1)$ For all $y$ in $\mathcal{X}$, we have the following.

$$
\left\|x_{n}-x, y\right\|=\left\|x_{n}-x+y, y\right\|=\left\|x_{n}-(x-y), x-(x-y)\right\| .
$$

Hence we have $\lim _{n \rightarrow \infty}\left\|x_{n}-x, y\right\|=0$ for all $y$ in $\mathcal{X}$.

Theorem 1.6 (cf. [4] Lemma 1.6). For a convergent sequence $\left\{x_{n}\right\}$ in a linear 2-normed space $\mathcal{X}$, we have $\lim _{n \rightarrow \infty}\left\|x_{n}, y\right\|=\|x, y\|=$ $\left\|\lim _{n \rightarrow \infty} x_{n}, y\right\|$ for all $y \in \mathcal{X}$.

Proof. In Lemma 1.5(2), take $a=0$.

The following lemma has fewer conditions than Lemma 1.2 of [4]. We need only two linearly independent vectors.

Lemma 1.7 (cf. [4] Lemma 1.2). Let $(\mathcal{X},\|\cdot, \cdot\|)$ be a linear 2-normed space with $\operatorname{dim} \mathcal{X}>1$. If $\|x, y\|=\|x, z\|=0$ for linearly independent $y, z \in \mathcal{X}$, then $x=0$.

In particular, If $\|x, y\|=0$ for all $y \in \mathcal{X}$, then $x=0$.

Proof. By the hypothesis, $x$ and $y$ are linearly dependent, and also $x$ and $z$ are linearly dependent. Then since $y$ and $z$ are not zero, there exist non-zero scalars $\alpha$ and $\alpha^{\prime}$ such that $\alpha x+\beta y=0$ and $\alpha^{\prime} x+\beta^{\prime} z=0$ for some scalars $\beta$ and $\beta^{\prime}$. Hence we have

$$
x=-\frac{\beta}{\alpha} y \text { and } x=-\frac{\beta^{\prime}}{\alpha^{\prime}} z .
$$


Thus we have

$$
-\frac{\beta}{\alpha} y+\frac{\beta^{\prime}}{\alpha^{\prime}} z=0 .
$$

Since $y$ and $z$ are linearly independent, we have $\beta=\beta^{\prime}=0$. Therefore we have $x=0$.

\section{Main results}

Firstly, we define the 2-metric space.

Definition 2.1. A 2-metric space is a space $\mathcal{X}$ with a real-valued nonnegative function $d$ defined on $\mathcal{X} \times \mathcal{X} \times \mathcal{X}$ which the following conditions:

(2M1) For each pair of elements $x, y$ in $\mathcal{X}$ with $x \neq y$, there exists an element $z$ in $\mathcal{X}$ such that $d(x, y, z) \neq 0$,

(2M2) $d(x, y, z)=0$ whenever at least two of the points $x, y, z$ are equal,

(2M3) $d(x, y, z)=d(x, z, y)=d(y, z, x)$,

$(2 \mathrm{M} 4) d(x, y, z) \leq d(x, y, w)+d(x, w, z)+d(w, y, z)$,

for all $x, y, z, w$ in $\mathcal{X}$. $d$ is called a 2-metric the space $\mathcal{X}$ and $(\mathcal{X}, d)$ is called a 2-metric space.

From the condition (2M3), we can easily show that $d(x, y, z)=d(x, z, y)$ $=d(y, z, x)=d(y, x, z)=d(z, x, y)=d(z, y, x)$.

If $(\mathcal{X}, d)$ is a linear 2-normed space, then the function $d(x, y, z)=$ $\|x-z, y-z\|$ defines a 2 -metric on $\mathcal{X}$. Therefore every 2-normed space will be considered to be a 2-metric space with the 2-metric defined in this sense.

Three or more points $p_{1}, p_{2}, p_{3}, \cdots$ are said to be collinear if they lie on a single straight line, that is, for each $i=3,4,5, \cdots$, if $p_{1} \neq p_{2}$ and $p_{1} \neq p_{i}$, then there is a real number $t_{i}$ such that $p_{1}-p_{2}=t_{i}\left(p_{1}-p_{i}\right)$.

Definition 2.2. A point $p$ in a linear 2 -normed space $\mathcal{X}$ is called 2-norm midpoint of 3 non-collinear points $x, y, z$ in $\mathcal{X}$ if $d(x, y, p)=$ $d(x, p, z)=d(p, y, z)=\frac{1}{3} d(x, y, z)$.

For non-collinear points $x, y, z$ in $\mathcal{X}$, let $T(x, y, z)=\{w \in \mathcal{X}$ : $d(x, y, z)=d(x, y, w)+d(x, w, z)+d(w, y, z)\} . T(x, y, z)$ will be called the triangle with vertices $x, y$ and $z$. Furthermore, we will designate the area of $T(x, y, z)$ to be $d(x, y, z)$. A point $p$ of $\mathcal{X}$ will be a center of $T(x, y, z)$ if $p$ is a 2-norm midpoint of $x, y$ and $z$.

The following theorem was proved in [3]. We give another easy solutions. 
Theorem 2.3 ([3] THEOREM 2.1). Suppose that $\mathcal{X}$ is a linear 2normed space.

(1) $x \in T(a, b, c)$ if and only if $x-y \in T(a-y, b-y, c-y)$.

(2) $T(a+p, b+p, c+p)=T(a, b, c)+p$.

(3) For a real number $\alpha$, we have

$$
\alpha T(a, b, c)=T(\alpha a, \alpha b, \alpha c) .
$$

(4) Let a sequence $\left\{x_{n}\right\}$ in $\mathcal{X}$ converge to a point $x$ in $\mathcal{X}$. If $\left\{x_{n}\right\}$ is a sequence in $T(a, b, c)$ for some non-collinear points of $\mathcal{X}$, then $x$ is a point in $T(a, b, c)$.

Proof. (1) Suppose that $x \in T(a, b, c)$. Then we have

$$
d(a, b, c)=d(a, b, x)+d(a, x, c)+d(x, b, c)
$$

or

$$
\|a-b, a-c\|=\|a-x, b-x\|+\|b-x, c-x\|+\|c-x, a-x\|
$$

if and only if

$$
\begin{aligned}
\| a- & y-(b-y), a-y-(c-y) \| \\
= & \|a-y-(x-y), b-y-(x-y)\| \\
& \quad+\|b-y-(x-y), c-y-(x-y)\| \\
& \quad+\|c-y-(x-y), a-y-(x-y)\|
\end{aligned}
$$

for all $y \in \mathcal{X}$. Therefore we have $x-y \in T(a-y, b-y, c-y)$.

(2) Suppose that $x \in T(a+p, b+p, c+p)$. Then we have

$$
\begin{aligned}
& d(a+p, b+p, c+p) \\
& =d(a+p, b+p, x)+d(a+p, x, c+p)+d(x, b+p, c+p)
\end{aligned}
$$

or

$$
\begin{aligned}
& \|a+p-(b+p), a+p-(c+p)\| \\
& =\|a+p-x, b+p-x\| \\
& \quad+\|b+p-x, c+p-x\|+\|c+p-x, a+p-x\|
\end{aligned}
$$

if and only if

$$
\begin{aligned}
\|a-b, a-c\|= & \|a-(x-p), b-(x-p)\| \\
& +\|b-(x-p), c-(x-p)\| \\
& +\|c-(x-p), a-(x-p)\| .
\end{aligned}
$$

Therefore we have $x-p \in T(a, b, c)$ or $x \in T(a, b, c)+p$. 
(3) We may assume that $\alpha$ is not zero. For all $y \in \alpha T(a, b, c)$, there is a point $x \in T(a, b, c)$ such that $y=\alpha x$. Therefore we have

$$
d(a, b, c)=d(a, b, x)+d(a, x, c)+d(x, b, c)
$$

or

$$
\|a-b, a-c\|=\|a-x, b-x\|+\|b-x, c-x\|+\|c-x, a-x\| .
$$

Hence from multiplying both sides by $|\alpha|^{2}$, we have

$$
\begin{aligned}
& \|\alpha a-\alpha b, \alpha a-\alpha c\| \\
& =\|\alpha a-\alpha x, \alpha b-\alpha x\|+\|\alpha b-\alpha x, \alpha c-\alpha x\|+\|\alpha c-\alpha x, \alpha a-\alpha x\| .
\end{aligned}
$$

Thus we have $y=\alpha x \in T(\alpha a, \alpha b, \alpha c)$.

On the other hand, for all $x \in T(\alpha a, \alpha b, \alpha c)$, we have

$$
d(\alpha a, \alpha b, \alpha c)=d(\alpha a, \alpha b, x)+d(\alpha a, x, \alpha c)+d(x, \alpha b, \alpha c)
$$

or

$$
\begin{aligned}
& \|\alpha a-\alpha b, \alpha a-\alpha c\| \\
& =\|\alpha a-x, \alpha b-x\|+\|\alpha b-x, \alpha c-x\|+\|\alpha c-x, \alpha a-x\| .
\end{aligned}
$$

Hence from dividing both sides by $|\alpha|^{2}$, we have

$$
\|a-b, a-c\|=\left\|a-\frac{x}{\alpha}, b-\frac{x}{\alpha}\right\|+\left\|b-\frac{x}{\alpha}, c-\frac{x}{\alpha}\right\|+\left\|c-\frac{x}{\alpha}, a-\frac{x}{\alpha}\right\| .
$$

Thus we have $\frac{x}{\alpha} \in T(a, b, c)$ or $x \in \alpha T(a, b, c)$.

(4) Assume that $x_{n} \in T(a, b, c)$ and $x_{n} \rightarrow x$ as $n \rightarrow \infty$. Then we have $d(a, b, c)=d\left(a, b, x_{n}\right)+d\left(a, x_{n}, c\right)+d\left(x_{n}, b, c\right)$. Since $d\left(a, b, x_{n}\right)=$ $\left\|a-x_{n}, b-x_{n}\right\|$, by lemma 1.5 we have $\lim _{n \rightarrow \infty} d\left(a, b, x_{n}\right)=d(a, b, x)$. Hence we have

$$
\begin{aligned}
d(a, b, c) & =\lim _{n \rightarrow \infty} d(a, b, c) \\
& =\lim _{n \rightarrow \infty}\left(d\left(a, b, x_{n}\right)+d\left(a, x_{n}, c\right)+d\left(x_{n}, b, c\right)\right) \\
& =d(a, b, x)+d(a, x, c)+d(x, b, c) .
\end{aligned}
$$

Therefore $x \in T(a, b, c)$.

Definition 2.4. Let $\mathcal{X}$ be a linear 2-normed space. For two points $b, c$ in $\mathcal{X}$, let $E(b, c)=\{x \in \mathcal{X}:\|x, b+c\|=\|x, b\|+\|x, c\|\}$. We will call $E(b, c)(=E(c, b))$ the 2-norm equality with respect to $b$ and $c$.

If the set $\{x, b\}$ or the set $\{x, c\}$ is linearly dependent, then $x \in$ $E(b, c)$. Hence $E(b, c)$ is a non-empty set. 
Theorem 2.5. Let $b$ and $c$ be points in a linear 2-normed space $\mathcal{X}$. Then we have the following.

(1) For all $b \in \mathcal{X}$ and non-negative real number $\alpha$, we have $E(b, \alpha b)=$ $\mathcal{X}$. For all non-zero $b \in \mathcal{X}$ and negative real number $\alpha$, we have $E(b, \alpha b)=\{\beta b: \beta \in \mathbb{R}\}$.

(2) For all non-zero $\alpha \in \mathbb{R}$ and $x \in E(b, c)$, we have

$E(b, c)=\alpha E(b, c)=E(\alpha b, \alpha c)=E(b+\alpha x, c)=E(b, c+\alpha x)$.

(3) If $\|x, b+c\|=0$ for a non-zero point $x \in E(b, c)$, then $x, b$ and $c$ are pairwise linearly dependent.

Therefore if $b$ and $c$ are linearly independent and a non-zero point $x \in E(b, c)$, then $\|x, b+c\| \neq 0$ or $x$ and $b+c$ are linearly independent.

(4) The points $b$ and $c$ are linearly dependent if and only if $E(b, c)$ is a subspace of $\mathcal{X}$.

In this case, the dimension of $E(b, c)$ over $\mathbb{R}$ is 1 or $\operatorname{dim} \mathcal{X}$.

(5) A sequence $\left\{x_{n}\right\}$ in $\mathcal{X}$ converges to a point $x$ in $\mathcal{X}$. If $\left\{x_{n}\right\}$ is a sequence in $E(b, c)$, then $x$ is a point in $E(b, c)$.

Proof. (1) For all $x \in \mathcal{X}$, we have $\|x, b+\alpha b\|=(1+\alpha)\|x, b\|=$ $\|x, b\|+\|x, \alpha b\|$. Therefore we have $E(b, \alpha b)=\mathcal{X}$.

Next suppose that $\alpha$ is a negative real number and $x \in E(b, \alpha b)$. Assume that $x$ and $b$ are linearly independent. Then we have

$$
|1+\alpha|\|x, b\|=\|x, b+\alpha b\|=\|x, b\|+\|x, \alpha b\|=(1+|\alpha|)\|x, b\| .
$$

Since $\|x, b\| \neq 0$, we have $|1+\alpha|=1+|\alpha|$.

In case $-1 \leq \alpha<0$, we have $1+\alpha=1-\alpha$ or $\alpha=0$. This is a contradiction. The other case $\alpha<-1$, we have $-1-\alpha=1-\alpha$ or $-1=1$. These contradictions imply that $x$ and $b$ are linearly dependent. Since $b$ is not zero, there is a real number $\beta_{0}$ such that $x=\beta_{0} b$. Thus $E(b, \alpha b) \subset\{\beta b: \beta \in \mathbb{R}\}$.

On the other hand, for all $\beta \in \mathbb{R}$ and all $b \in \mathcal{X}$, we have $\|\beta b, b+\alpha b\|=$ $0=\|\beta b, b\|+\|\beta b, \alpha b\|$. Thus $\{\beta b: \beta \in \mathbb{R}\} \subset E(b, \alpha b)$. Therefore we have $E(b, \alpha b)=\{\beta b: \beta \in \mathbb{R}\}$.

(2) For all $x \in E(b, c)$, we have

$$
\begin{aligned}
& \mid x, b+c\|=\| x, b\|+\| x, c \| \\
\Leftrightarrow & \left\|\alpha^{-1} x, b+c\right\|=\left\|\alpha^{-1} x, b\right\|+\left\|\alpha^{-1} x, c\right\| \\
\Leftrightarrow & \|x, \alpha(b+c)\|=\|x, \alpha b\|+\|x, \alpha c\| \\
\Leftrightarrow & \|x, b+\alpha x+c\|=\|x, b+\alpha x\|+\|x, c\|
\end{aligned}
$$




$$
\Leftrightarrow \quad\|x, b+c+\alpha x\|=\|x, b\|+\|x, c+\alpha x\| .
$$

Therefore we have

$$
E(b, c)=\alpha E(b, c)=E(\alpha(b, c))=E(b+\alpha x, c)=E(b, c+\alpha x) .
$$

(3) By the hypothesis we have $0=\|x, b+c\|=\|x, b\|+\|x, c\|$. Hence we have $0=\|x, b\|=\|x, c\|$. Therefore $x, b$ and $c$ are pairwise linearly dependent by $(2 \mathrm{~N} 1)$ and Lemma 1.7 .

(4) Let $b$ and $c$ be linearly dependent. Then $E(b, c)$ is $\mathcal{X}$ or $\{\beta b: \beta \in$ $\mathbb{R}\}$ by $(1)$. Hence $E(b, c)$ is a subspace of $\mathcal{X}$.

On the other hand, let $E(b, c)$ be a subspace of $\mathcal{X}$. Since $b, c \in E(b, c)$, we have $b+c \in E(b, c)$. Then we have

$$
0=\|b+c, b+c\|=\|b+c, b\|+\|b+c, c\|=2\|b, c\| .
$$

Hence $b$ and $c$ are linearly dependent.

(5) Assume that $x_{n} \in E(b, c)$ and $x_{n} \rightarrow x$ as $n \rightarrow \infty$. Then we have $\left\|x_{n}, b+c\right\|=\left\|x_{n}, b\right\|+\left\|x_{n}, c\right\|$.

By lemma 1.5 we have

$$
\begin{aligned}
\|x, b+c\| & =\lim _{n \rightarrow \infty}\left\|x_{n}, b+c\right\| \\
& =\lim _{n \rightarrow \infty}\left(\left\|x_{n}, b\right\|+\left\|x_{n}, c\right\|\right) \\
& =\|x, b\|+\|x, c\|
\end{aligned}
$$

Therefore we have $x \in E(b, c)$.

\section{References}

[1] S. Gähler, 2-metrische Räume und ihre topologische Struktur, Math. Nachr. 26 (1963), 115-148.

[2] S. Gähler, Lineare 2-normierte Räumen, Math. Nachr. 28 (1964), 1-43.

[3] A. White Jr. and Y. J. Cho, Linear mapping on linear 2-normed spaces, Bull. Korean Math. Soc. 21 (1984), no. 1, 1-6.

[4] W. G. Park, Approximate additive mapping in 2-Banach spaces and related topics, J. Math. Anal. Appl. 376 (2011), 193-202.

*

Department of Mathematics Education

Mokwon University

Daejeon 302-729, Republic of Korea

E-mail: math888@naver.com 